\title{
Experimental and Theoretical Analysis for a Fluid-Loaded, Simply Supported Plate Covered by a Damping and Decoupling Composite Acoustic Coating
}

\author{
Baihua Yuan, ${ }^{1}$ Meng Chen, ${ }^{2}$ Yu Liu, ${ }^{2}$ Shexu Zhao, ${ }^{1}$ and Heng Jiang ${ }^{2}$ \\ ${ }^{1}$ Department of Engineering Mechanics, School of Naval Architecture, Ocean \& Civil Engineering, Shanghai Jiaotong University, \\ Minhang, Shanghai 200240, China \\ ${ }^{2}$ Key Laboratory of Microgravity, Institute of Mechanics, Chinese Academy of Sciences, Beijing 100190, China
}

Correspondence should be addressed to Baihua Yuan; jxaj@163.com and Heng Jiang; hengjiang@imech.ac.cn

Received 23 September 2016; Revised 13 December 2016; Accepted 22 December 2016; Published 6 February 2017

Academic Editor: Miguel Neves

Copyright (C) 2017 Baihua Yuan et al. This is an open access article distributed under the Creative Commons Attribution License, which permits unrestricted use, distribution, and reproduction in any medium, provided the original work is properly cited.

\begin{abstract}
This work presents a vibroacoustic response model for a fluid-loaded, simply supported rectangular plate covered by a composite acoustic coating consisting of damping and decoupling layers. The model treated the damping layer and base plate as a unified whole under pure bending moments and the decoupling layer as a three-dimensional, isotropic, linear elastic solid. The validity of the model was verified by both numerical analysis and experiments and was shown to accurately extend previous studies that were limited to a plate covered by a single damping or decoupling layer with an evaluation confined solely to numerical analysis. The trends of the numerical and experimental results are generally consistent, with some differences due to the influences of water pressure and the frequency dependence of the material parameters, which are not taken into account by the numerical analysis. Both experimental and numerical results consistently show that the radiated noise reduction effect of the composite coating is superior to that of single-type coatings, which is attributed to the fact that the composite coating combines the merits of both the high vibration suppression performance of the damping layer and the superior vibration isolation performance of the decoupling layer.
\end{abstract}

\section{Introduction}

As an effective noise reduction approach for resonant structures immersed in water, the addition of a composite acoustic coating consisting of both damping and decoupling layers is proposed herein as a new method to reduce the vibrations and radiated noise generated in the structure. Such a composite acoustic coating combines the high vibration suppression performance of a damping layer and the superior vibration isolation performance of a decoupling layer, which can dramatically reduce vibrations and radiated noise with negligible increase in the thickness or weight of the acoustic coating.

A damping coating suppresses structural vibrations and reduces radiated noise through the internal friction of a viscoelastic polymer chain. Numerous studies have been performed regarding the vibroacoustics of resonant structures covered by damping coatings. Alam and Asnani $[1,2]$ employed variational principles to derive the governing equation of a cylindrical shell covered by a multilayered damping coating. Laulagnet and Guyader [3-5] researched the vibroacoustic response of a finite cylindrical shell covered by a damping coating based on Donnell and Flügge thin shell theory. Saravanan et al. [6-8] applied the finite element method to study the vibroacoustic response of a damped cylindrical shell.

A decoupling coating lessens the sound radiation by reducing vibration transfer from the resonant structure to the fluid via vibration isolation. Three types of dynamic models have been applied to decoupling coatings. The first of model is based on the theory of wave propagation in a layered media [9] and is derived using an impedance transfer matrix [10-12], which just regards the tensile stresses and longitudinal waves in the decoupling material. The second type is the locally reacting model [13-16], which assumes that 
the decoupling material behaves as a set of evenly distributed massless springs on the plate. Such models however remain limited by their inherent inability to capture the complex propagation of elastic waves in the decoupling material [17]. The last type of model employs the three-dimensional (3D) theory of elasticity. Compared with above two models, 3D elasticity models are more precise because the model takes into consideration thickness deformation and bending, in addition to shear and tensile stresses in the decoupling material. Ko and Keltie $[18,19]$ employed the 3D theory of elasticity for base plate/decoupling-coating systems of infinite dimensions. Berry et al. [17] derived an exact formulation using $3 \mathrm{D}$ elasticity to describe the vibroacoustic response of a finite, simply supported rectangular plate covered by a decoupling material and immersed in a heavy fluid. Xin [20] developed an exact elasticity model for rib-stiffened plates covered by multilayer decoupling acoustic coatings, which was built upon standard strain elasticity theory.

From the above discussion, we see that previously published studies have been concerned with resonant structures covered with single-layer damping or decoupling coatings or multilayered coatings comprising only damping layers and have provided no experimental verification of their theoretical-based results. Therefore, the vibroacoustic response of a resonant structure covered by a composite acoustic coating comprising both damping and decoupling layers is fairly unknown.

This paper proposes an exact analytical model for a fluid-loaded, simply supported rectangular plate covered by a damping and decoupling composite acoustic coating. The model treats the damping layer and base plate as a unified whole under pure bending moments and employs 3D elasticity theory for the decoupling layer. The vibration suppression performance, decoupling performance, and radiated noise reduction effect of various composite coatings were studied theoretically and experimentally, and the results reveal the mechanisms of noise reduction and provide principles for appropriate materials selection for the composite coating. The experiments also validate the proposed analytical model.

\section{Theoretical Analysis}

2.1. Dynamics of the System. As shown in Figure 1, the studied structure is a rectangular (dimensions $a \times b$ ), simply supported finite plate covered by a damping and decoupling composite acoustic coating, which is immersed in water. The damping layer $\left(\mathrm{H}_{2}\right)$ is bonded to the base plate $\left(H_{1}\right)$, whereas the decoupling layer $\left(\mathrm{H}_{3}\right)$ is bonded to the damping layer. The excitation is a point force exerted on the base plate at $x=x_{0}$ and $y=y_{0}$ in the vicinity of the plate corner. Because the damping layer is in contact with the base plate and has a similar Young's modulus, which is far greater than that of the decoupling layer, the damping layer and the base plate are considered as single unified body and are described using classical Love-Kirchhoff plate theory for pure bending moments, while the decoupling layer is treated as a linear, homogeneous, and isotropic elastic material and described using the 3D model presented by Berry et al. [17].
The equation of motion of the composite plate, consisting of the base plate and the damping layer, is given by

$$
\begin{aligned}
& {\left[\bar{B}\left(\frac{\partial^{2}}{\partial x^{2}}+\frac{\partial^{2}}{\partial y^{2}}\right)^{2}+\left(\rho_{1} H_{1}+\rho_{2} H_{2}\right) \frac{\partial^{2}}{\partial t^{2}}\right] w_{1}(x, y)} \\
& \quad=f(x, y)-\sigma_{z}(x, y, 0),
\end{aligned}
$$

where $\rho_{1}, \rho_{2}, H_{1}$, and $H_{2}$ are the density and the thickness of the base plate and the damping layer, respectively, $w_{1}$ is the transverse displacement of the composite plate, $f(x, y)$ is the external force per unit area acting on the plate, and $\sigma_{z}(x, y, 0)$ is the normal stress exerted by the decoupling layer on the damping layer. $\bar{B}$ represents the flexural stiffness of the entire composite plate and is derived as

$$
\begin{aligned}
\bar{B}= & \frac{E_{1}\left(H_{1}^{3}-3 H_{1}^{2} \zeta+3 H_{1} \zeta^{2}\right)}{3\left(1-\sigma_{1}^{2}\right)} \\
& +\frac{E_{2}\left(H_{2}^{3}+3 H_{2}^{2} \zeta+3 H_{2} \zeta^{2}\right)}{3\left(1-\sigma_{2}^{2}\right)},
\end{aligned}
$$

where $E_{1}, E_{2}, \sigma_{1}$, and $\sigma_{2}$ are the complex Young's modulus and the Poisson's ratio of the base plate and damping layer, respectively. $E_{2}$ is defined as $E_{2}=E_{2}^{\prime}+i E_{2}^{\prime \prime}=E_{2}^{\prime}\left(1+i \beta_{2}\right)$, where $\beta_{2}$ is the loss factor of the damping material, and $\zeta$ is the distance from the neutral axis of the base plate to the top of the damping layer and is given by

$$
\zeta=\frac{1}{2} \frac{\left(E_{1} /\left(1-\sigma_{1}\right)\right) H_{1}^{2}-\left(E_{2} /\left(1-\sigma_{2}\right)\right) H_{2}^{2}}{\left(E_{1} /\left(1-\sigma_{1}\right)\right) H_{1}+\left(E_{2} /\left(1-\sigma_{2}\right)\right) H_{2}} .
$$

According to the pure bending moment theory, (1) is valid only if the bending wavelength of the composite plate is greater than $6\left(H_{1}+H_{2}\right)$ since the shear deformations are caused by shear stresses acting on the cross section and the rotational inertia [21].

The decoupling layer is governed by the Navier-Cauchy equation:

$$
\rho_{3} \frac{\partial^{2} u}{\partial t^{2}}=\left(\lambda_{3}+\mu_{3}\right) \nabla(\nabla \cdot u)+\mu_{3} \nabla^{2} u
$$

where $\rho_{3}$ is the density, $\lambda_{3}$ and $\mu_{3}$ are the Lamé coefficients, and $u=\left(u_{3}, v_{3}, w_{3}\right)$ is the displacement in the decoupling layer. According to Achenbach [22], $u$ is divided into an irrotational wave and a rotational wave as

$$
u=\nabla \phi+\nabla \times \psi,
$$

with an additional relation of $\psi$ :

$$
\nabla \cdot \psi=0
$$

where $\phi$ is the scalar potential corresponding to a longitudinal wave and $\psi=\left(\psi_{x}, \psi_{y}, \psi_{z}\right)$ is the vector potential associated with a transverse wave. Both $\phi$ and $\psi$ are governed by the Helmholtz equations:

$$
\begin{aligned}
& \nabla^{2} \phi=\frac{1}{c_{L}} \frac{\partial^{2} \phi}{\partial t^{2}}, \\
& \nabla^{2} \psi=\frac{1}{c_{T}} \frac{\partial^{2} \psi}{\partial t^{2}},
\end{aligned}
$$




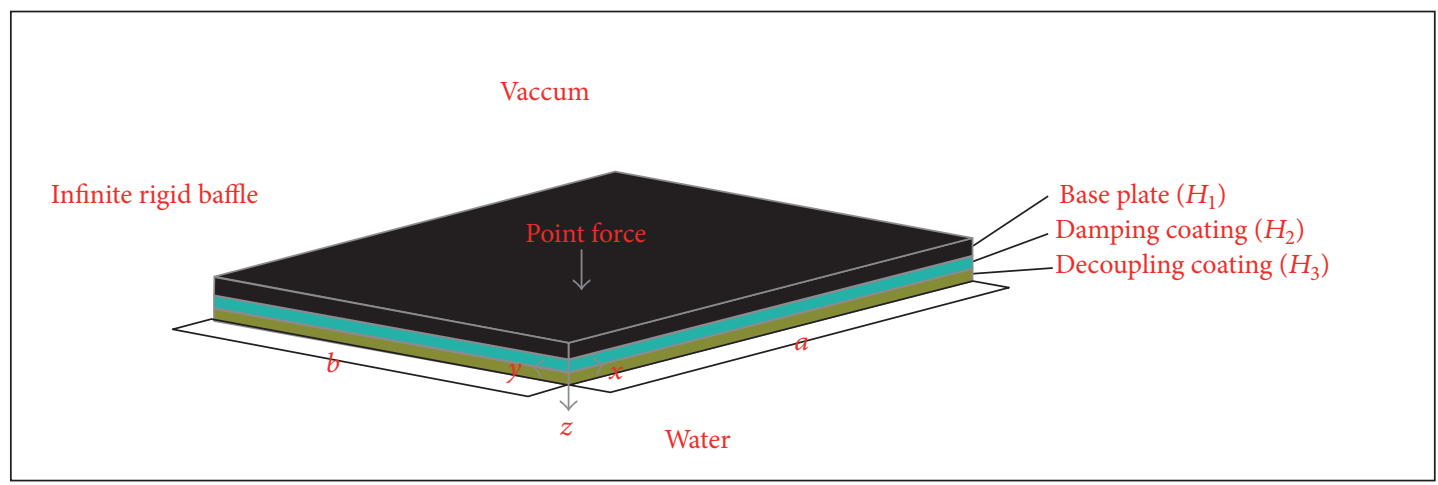

FIGURE 1: Schematic illustration of the resonant structure system.

where $c_{L}=\sqrt{\left(\lambda_{3}+\mu_{3}\right) / \rho_{3}}$ and $c_{T}=\sqrt{\mu_{3} / \rho_{3}}$ are the speed of the longitudinal wave and transverse wave, respectively. The stress components in the decoupling layer are obtained using the constitutive relation:

$$
\sigma_{i j}=\lambda_{3}(\nabla \cdot u) \delta_{i j}+\mu_{3}\left(u_{i, j}+u_{j, i}\right),
$$

in which $\delta_{i j}=1(i=j)$ or $\delta_{i j}=0(i \neq j)$.

Because the water media is considered ideal and nonviscous, the acoustic pressure $p$ radiated by the composite system is therefore governed by the wave equation:

$$
\nabla^{2} p-\frac{1}{c_{0}^{2}} \frac{\partial^{2} p}{\partial t^{2}}=0
$$

where $c_{0}$ is the speed of sound in the medium. According to the Kirchhoff-Helmholtz theorem, the acoustic pressure in the fluid half-space relates to the normal displacement at the coating surface by the Rayleigh integral [17]:

$$
\begin{aligned}
& p(x, y, z) \\
& \quad=\omega^{2} \rho_{0} \int_{0}^{a} \int_{0}^{b} w_{3}\left(x^{\prime}, y^{\prime}, H_{3}\right) \frac{e^{-j k_{0} R}}{2 \pi R} d x^{\prime} d y^{\prime},
\end{aligned}
$$

where $R=\sqrt{\left(x-x^{\prime}\right)^{2}+\left(y-y^{\prime}\right)^{2}+\left(z-H_{3}\right)^{2}}\left(z \geq H_{3}\right), \rho_{0}$ is the density of the medium, and $k_{0}=\omega / c_{0}$ is the wave number in the medium.

2.2. Boundary Conditions and Solutions. The simply supported boundary conditions at the edges of the base plate and the damping layer are

$$
\begin{aligned}
w_{1}=0 \quad \text { at } x=0, x=a, y=0, y=b ; \\
\frac{\partial^{2} w_{1}}{\partial y^{2}}=0 \quad \text { at } x=0, x=a ; \\
\frac{\partial^{2} w_{1}}{\partial x^{2}}=0 \quad \text { at } y=0, y=b .
\end{aligned}
$$

The boundary conditions at the damping-decoupling interface $(z=0)$ represent the continuity of displacements:

$$
\begin{array}{r}
w_{1}(x, y)=w_{3}(x, y, 0) ; \\
\left(\zeta+H_{2}\right) \frac{\partial w_{1}(x, y)}{\partial x}=u_{3}(x, y, 0) .
\end{array}
$$

The boundary conditions on the lateral sides of the decoupling layer are [17]

$$
\begin{aligned}
w_{3}=0 & \text { at } x=0, x=a, y=0, y=b, \\
u_{3}=0 & \text { at } y=0, y=b, \\
v_{3}=0 & \text { at } x=0, x=a, \\
\sigma_{x x}=0 & \text { at } x=0, x=a, y=0, y=b, \\
\sigma_{y y}=0 & \text { at } x=0, x=a, y=0, y=b, \\
\sigma_{z x}=0 & \text { at } y=0, y=b, \\
\sigma_{y z}=0 & \text { at } x=0, x=a .
\end{aligned}
$$

The above boundary conditions physically show that the lateral sides of the decoupling layer are fixed in the $z$ direction but are free to move along the direction normal to the side, and that will allow the scalar potential $\varphi$ and the potential vector $\psi$ to be simply expressed in terms of trigonometric functions.

The boundary conditions at the decoupling-fluid interface $\left(z=H_{3}\right)$ represent the continuity of stresses:

$$
\begin{aligned}
\left.\frac{\partial^{2} w_{3}}{\partial t^{2}}\right|_{z=H_{3}} & =\left.\frac{1}{\rho_{0}} \frac{\partial p}{\partial z}\right|_{z=H_{3}}, \\
\sigma_{z z}\left(x, y, H_{3}\right) & =-p\left(x, y, H_{3}\right), \\
\sigma_{z x}\left(x, y, H_{3}\right) & =0, \\
\sigma_{y z}\left(x, y, H_{3}\right) & =0 .
\end{aligned}
$$

The problem is entirely defined using (1)-(14). In the following, solutions are derived for the four unknown variables 
$w_{1}, \phi, \psi_{x}$, and $\psi_{y}$. The solution of (1) can be written in terms of a Fourier series:

$$
w_{1}(x, y)=\sum_{m=1}^{\infty} \sum_{n=1}^{\infty} w_{1, m n} \sin \left(\frac{m \pi x}{a}\right) \sin \left(\frac{n \pi y}{b}\right)
$$

The solutions of the Helmholtz equations (see (7)) can also be written in terms of a Fourier series:

$$
\begin{aligned}
\varphi(x, y, z) & =\sum_{p=1}^{\infty} \sum_{q=1}^{\infty} \varphi_{p q}(z) \sin \left(\frac{p \pi x}{a}\right) \sin \left(\frac{q \pi y}{b}\right), \\
\psi_{x}(x, y, z) & =\sum_{p=1}^{\infty} \sum_{q=0}^{\infty} \psi_{x, p q}(z) \sin \left(\frac{p \pi x}{a}\right) \cos \left(\frac{q \pi y}{b}\right), \\
\psi_{y}(x, y, z) & =\sum_{p=0}^{\infty} \sum_{q=1}^{\infty} \psi_{x, p q}(z) \cos \left(\frac{p \pi x}{a}\right) \sin \left(\frac{q \pi y}{b}\right) .
\end{aligned}
$$

The choices of such trigonometric functions are determined by the boundary conditions of (13), in that the displacements and stress components on the lateral sides of the decoupling layer derived from these trigonometric functions will automatically satisfy the boundary conditions of (13). Substituting (16), (17), and (18) into (7) results in the following equations:

$$
\begin{array}{r}
K_{L, p q} \varphi_{p q}(z)+\frac{\partial^{2} \varphi_{p q}(z)}{\partial z^{2}}=0, \\
K_{T, p q} \psi_{x, p q}(z)+\frac{\partial^{2} \psi_{x, p q}(z)}{\partial z^{2}}=0, \\
K_{T, p q} \psi_{y, p q}(z)+\frac{\partial^{2} \psi_{y, p q}(z)}{\partial z^{2}}=0,
\end{array}
$$

where

$$
\begin{aligned}
& K_{L, p q}=\sqrt{\left[\frac{\omega^{2}}{c_{L}^{2}}-\left(\frac{p \pi}{a}\right)^{2}-\left(\frac{q \pi}{b}\right)^{2}\right]}, \\
& K_{T, p q}=\sqrt{\left[\frac{\omega^{2}}{c_{T}^{2}}-\left(\frac{p \pi}{a}\right)^{2}-\left(\frac{q \pi}{b}\right)^{2}\right]} .
\end{aligned}
$$

The solutions of (19) can be readily given as

$$
\begin{gathered}
\varphi_{p q}(z)=S_{p q} \cos \left(K_{L, p q} z\right)+T_{p q} \sin \left(K_{L, p q} z\right), \\
\psi_{x, p q}(z)=U_{x, p q} \cos \left(K_{T, p q} z\right)+V_{x, p q} \sin \left(K_{T, p q} z\right), \\
\psi_{y, p q}(z)=U_{y, p q} \cos \left(K_{T, p q} z\right)+V_{y, p q} \sin \left(K_{T, p q} z\right),
\end{gathered}
$$

where $S_{p q}, T_{p q}, U_{x, p q}, V_{x, p q}, U_{y, p q}$, and $V_{y, p q}$ are unknown coefficients related to the longitudinal wave and the shear wave in the decoupling layer. By substituting (16), (17), and (18) into (5) and (8), the corresponding displacements and stresses can be obtained, respectively, as

$$
\begin{aligned}
& u_{3}(x, y, z)=\frac{\partial \varphi}{\partial x}+\frac{\partial \psi_{z}}{\partial y}-\frac{\partial \psi_{y}}{\partial z}=\sum_{p=0}^{\infty} \sum_{q=1}^{\infty} \cos \left(\frac{p \pi x}{a}\right) \\
& \cdot \sin \left(\frac{q \pi y}{b}\right)\left(\frac{p \pi}{a} \varphi_{p q}(z)-\frac{q \pi}{b} \psi_{z, p q}(z)\right. \\
& \left.-\frac{\partial \psi_{y, p q}(z)}{\partial z}\right) \\
& v_{3}(x, y, z)=\sum_{p=1}^{\infty} \sum_{q=0}^{\infty} v_{3, p q}(z) \sin \left(\frac{p \pi x}{a}\right) \cos \left(\frac{q \pi y}{b}\right) \\
& =\sum_{p=1}^{\infty} \sum_{q=0}^{\infty} \sin \left(\frac{p \pi x}{a}\right) \cos \left(\frac{q \pi y}{b}\right)\left(\frac{q \pi}{b} \varphi_{p q}(z)\right. \\
& \left.+\frac{p \pi}{a} \psi_{z, p q}(z)+\frac{\partial \psi_{x, p q}(z)}{\partial z}\right) \\
& w_{3}(x, y, z)=\frac{\partial \varphi}{\partial z}+\frac{\partial \psi_{y}}{\partial x}-\frac{\partial \psi_{x}}{\partial y}=\sum_{p=1}^{\infty} \sum_{q=0}^{\infty} \sin \left(\frac{p \pi x}{a}\right) \\
& \cdot \sin \left(\frac{q \pi y}{b}\right)\left(\frac{\partial \varphi_{p q}(z)}{\partial z}-\frac{p \pi}{a} \psi_{y, p q}(z)\right. \\
& \left.+\frac{q \pi}{b} \psi_{x, p q}(z)\right) \\
& \sigma_{z}(x, y, z)=\sum_{p=1}^{\infty} \sum_{q=1}^{\infty} \sin \left(\frac{p \pi x}{a}\right) \sin \left(\frac{q \pi y}{b}\right) \\
& \cdot\left(-\frac{p \pi}{a} \lambda_{3} u_{3, p q}(z)-\frac{q \pi}{b} \lambda_{3} v_{3, p q}(z)\right. \\
& \left.+\left(\lambda_{3}+2 \mu_{3}\right) \frac{\partial w_{3, p q}(z)}{\partial z}\right) \\
& \sigma_{y z}(x, y, z)=\sum_{p=1}^{\infty} \sum_{q=0}^{\infty} \sin \left(\frac{p \pi x}{a}\right) \cos \left(\frac{q \pi y}{b}\right) \\
& \cdot\left(\mu_{3} \frac{\partial v_{3, p q}(z)}{\partial z}+\mu_{3} \frac{q \pi}{b} w_{3, p q}(z)\right) \\
& \sigma_{z x}(x, y, z)=\sum_{p=0}^{\infty} \sum_{q=1}^{\infty} \cos \left(\frac{p \pi x}{a}\right) \sin \left(\frac{q \pi y}{b}\right) \\
& \cdot\left(\mu_{3} \frac{\partial u_{3, p q}(z)}{\partial z}+\mu_{3} \frac{p \pi}{a} w_{3, p q}(z)\right) \text {. }
\end{aligned}
$$


Substituting (15) and (25) into (1) results in the following equation:

$$
\begin{aligned}
\frac{a b}{4} & \left(\rho_{1} H_{1}+\rho_{2} H_{2}\right)\left(\omega_{m n}^{2}-\omega^{2}\right) w_{1, m n} \\
& =-F_{0} \sin \left(\frac{m \pi x_{0}}{a}\right) \sin \left(\frac{n \pi y_{0}}{b}\right)-\frac{a b}{4} \sigma_{z, m n}(0),
\end{aligned}
$$

where

$$
\begin{aligned}
\sigma_{z, m n}(0)= & -\left[\lambda_{3}\left(\frac{\omega}{c_{L}}\right)^{2}+2 \mu_{3} K_{L, m n}^{2}\right] S_{m n} \\
& -2 \mu_{3} K_{T, m n}^{2}\left(\frac{m \pi}{a}\right) V_{y, m n} \\
& +2 \mu_{3} K_{T, m n}\left(\frac{n \pi}{b}\right) V_{x, m n} .
\end{aligned}
$$

The term $\omega_{m n}=\sqrt{\left(\bar{B} /\left(\rho_{1} H_{1}+\rho_{1} H_{1}\right)\right)}\left[(m \pi / a)^{2}+(n \pi / b)^{2}\right]$ represents the natural angular frequencies of the base plate and damping layer composite. Substituting (15), (22), (23), and (24) into (12) results in the following equations:

$$
\begin{aligned}
w_{1, m n} & =K_{L, m n} T_{m n}-\left(\frac{m \pi}{a}\right) U_{y, m n}+\left(\frac{n \pi}{b}\right) U_{x, m n} \\
(\zeta+ & \left.H_{2}\right) \frac{m \pi}{a} K_{T, m n} w_{1, m n} \\
= & \frac{m \pi}{a} K_{T, m n} S_{m n}-\left(\frac{m \pi}{a}\right)\left(\frac{n \pi}{b}\right) V_{x, m n} \\
& -\left[\left(\frac{n \pi}{b}\right)^{2}+K_{T, m n}^{2}\right] V_{y, m n} \\
(\zeta+ & \left.H_{2}\right) \frac{n \pi}{b} K_{T, m n} w_{1, m n} \\
= & \frac{n \pi}{b} K_{T, m n} S_{m n}+\left[\left(\frac{m \pi}{a}\right)^{2}+K_{T, m n}^{2}\right] V_{x, m n} \\
+ & \left(\frac{m \pi}{a}\right)\left(\frac{n \pi}{b}\right) V_{y, m n} .
\end{aligned}
$$

$$
Z_{m n p q}=j \omega \rho_{0} \int_{0}^{a} \int_{0}^{b} \int_{0}^{a} \int_{0}^{b} \sin \left(\frac{m \pi x^{\prime}}{a}\right) \sin \left(\frac{n \pi y^{\prime}}{b}\right) \frac{e^{-j k_{0} R_{H_{3}}}}{2 \pi R_{H_{3}}} \sin \left(\frac{p \pi x}{a}\right) \sin \left(\frac{q \pi y}{b}\right) d x d y d x^{\prime} d y^{\prime} .
$$

Equations (28), (30), (31), (32), and (33) form the equations of the problem in terms of the unknown coefficients $w_{1, m n}, S_{m n}, T_{m n}, U_{x, m n}, V_{x, m n}, U_{y, m n}$, and $V_{y, m n}$. By simplifying
Substituting (24) and (25) into (14) results in the following equations:

$$
\begin{aligned}
& {\left[\left(\frac{\omega}{c_{L}}\right)^{2} \lambda_{3}+2 \mu_{3} K_{L, p q}^{2}\right] S_{p q} \cos \left(K_{L, p q} H_{3}\right)-\left[\left(\frac{\omega}{c_{L}}\right)^{2}\right.} \\
& \left.\cdot \lambda_{3}+2 \mu_{3} K_{L, p q}^{2}\right] T_{p q} \sin \left(K_{L, p q} H_{3}\right)-\frac{2 b}{q \pi} \\
& \quad \cdot \mu_{3} K_{T, p q}\left[\left(\frac{p \pi}{a}\right)^{2}+\left(\frac{q \pi}{b}\right)^{2}\right] U_{x, p q} \sin \left(K_{T, p q} H_{3}\right) \\
& \quad-\frac{2 b}{q \pi} \mu_{3} K_{T, p q}\left[\left(\frac{p \pi}{a}\right)^{2}+\left(\frac{q \pi}{b}\right)^{2}\right] V_{x, p q} \\
& \quad \cdot \cos \left(K_{T, p q} H_{3}\right)+\frac{2 j \omega}{a b}\left(\frac{\omega}{c_{T}}\right)^{2} \sum_{m=1}^{\infty} \sum_{n=1}^{\infty}\left(\frac{b}{n \pi}\right) \\
& \quad \cdot\left[U_{x, m n} \cos \left(K_{T, m n} H_{3}\right)-V_{x, m n} \sin \left(K_{T, m n} H_{3}\right)\right] \\
& \quad \cdot Z_{m n p q}=0, \\
& \left.\quad \cdot \frac{m \pi}{a}\right)\left[K_{L, m n} S_{m n} \sin \left(K_{L, m n} H_{3}\right)+K_{L, m n} T_{m n}\right. \\
& \left.\quad \cdot \cos \left(K_{T, m n} H_{3}\right)-V_{y, m n} \sin \left(K_{T, m n} H_{3}\right)\right] \\
& \left.\quad \cdot \cos \left(K_{L, m n} H_{3}\right)\right]=0,\left[U_{x, m n} \cos \left(K_{T, m n} H_{3}\right)\right. \\
& \left.\quad \cdot \cos \left(K_{L, m n} H_{3}\right)\right]+2\left(\frac{m \pi}{a}\right)\left(\frac{n \pi}{b}\right)\left[U_{x, m n}\right. \\
& \left.\quad+K_{L, m n} H_{3, m n} \cos \left(K_{T, m n} H_{3}\right)-V_{y, m n} \sin \left(K_{L, m n} H_{3}\right)+K_{L, m n} T_{m n} \sin \left(K_{T, m n} H_{3}\right)\right]+\left[\frac{\omega}{c_{T}}\right)^{2}
\end{aligned}
$$

where $Z_{\text {mnpq }}$ are the sound radiation impedance terms defined by (34) and can be computed using the integral transform techniques described by Foin et al. [14] and He [23]: the above equations, they can be written in a global matrix notation:

$$
\widetilde{A} \widetilde{X}=\widetilde{B}
$$


where

$$
\begin{aligned}
& \widetilde{A}=\left[\begin{array}{ccccccc}
0 & {[A]} & 0 & 0 & {[B]} & 0 & 0 \\
{[C]} & 0 & {[D]} & {[E]} & 0 & 0 & 0 \\
0 & {[F]} & {[G]} & {[H]} & {[I]} & 0 & 0 \\
0 & {[J]} & {[K]} & {[L]} & {[M]} & 0 & 0 \\
0 & {[N]} & {[O]} & {[P]} & {[Q]} & 0 & 0 \\
0 & 0 & 0 & {[R]} & 0 & {[S]} & 0 \\
0 & 0 & 0 & 0 & {[T]} & 0 & {[U]}
\end{array}\right], \\
& \widetilde{X}=\left\{\left\{w_{1, m n}\right\}^{T}\left\{S_{m n}\right\}^{T}\left\{T_{m n}\right\}^{T}\left\{U_{x, m n}\right\}^{T}\left\{V_{x, m n}\right\}^{T}\left\{U_{y, m n}\right\}^{T}\left\{V_{y, m n}\right\}^{T}\right\}^{T}, \\
& \widetilde{B}=\left\{\left\{-n \pi\left(\zeta+H_{2}\right) f_{m n}\right\}^{T} 0^{T} \quad 0^{T} \quad 0^{T} \quad 0^{T} \quad 0^{T} \quad 0^{T}\right\}^{T} .
\end{aligned}
$$

The solution of the unknown coefficients can then be obtained by solving the equation:

$$
\widetilde{X}=\widetilde{A}^{-1} \widetilde{B} .
$$

Once these coefficients are known, the displacement fields, the stress fields, and the acoustic pressure fields can be obtained.

In this paper, three vibroacoustic indicators are introduced: the mean squared velocity of the base plate $\left(v_{1}^{2}\right)$, the mean squared velocity of the outer surface of the decoupling layer $\left(v_{2}^{2}\right)$, and the radiated sound power by the system in water $(W)$. The definitions of the vibroacoustic indicators have been previously published $[14,15,17]$.

\section{Numerical Results}

3.1. Validation of the Proposed Formulation. The proposed model treated the damping layer and base plate as a unified whole under pure bending moments, as described by (1), the validity and effective frequency range of which have been widely studied and validated. In addition, the decoupling layer is treated as a linear, homogeneous, and isotropic elastic material and is described using the $3 \mathrm{D}$ model presented by Berry et al. [17]. To validate the proposed model, it is assumed that the thickness of the damping layer is zero, such that the model can be simplified to a vibroacoustic response for a simply supported rectangular plate covered by a single decoupling layer, as conducted previously [17]. Figure 2 shows the radiated sound power of the modeled system compared with that obtained in [17]. From Figure 2, the current results are found to match very well with those in [17].

3.2. Vibroacoustic Response with Different Composite Specimens. To investigate the noise reduction mechanism of the damping and decoupling composite acoustic coating, numerical calculations were performed based on three specimens with different decoupling layer parameters, which represent a varying influence on the vibroacoustic behavior. The structural dimensions and material properties of the coating are tabulated in Table 1 . The other parameters are given as follows.
A steel plate $\left[\rho_{1}=7850 \mathrm{~kg} / \mathrm{m}^{3}, E_{1}=2.1 \times 10^{11} \mathrm{~Pa}\right.$, and $\sigma_{1}=$ 0.3 ] of dimensions $a=0.78 \mathrm{~m}$ and $b=0.58 \mathrm{~m}$ and thickness $H_{1}=0.024 \mathrm{~m}$ is considered. The speed of sound and density of water are $c_{0}=1460 \mathrm{~m} / \mathrm{s}$ and $\rho_{0}=1000 \mathrm{~kg} / \mathrm{m}^{3}$, respectively. A point force is applied on the base plate at $x_{0}=0.195 \mathrm{~m}$ and $y_{0}=0.145 \mathrm{~m}$ relative to the plate corner.

Because (1) is valid only if the bending wavelength of the composite plate is greater than $6\left(H_{1}+H_{2}\right)$ [21], the upper limit on the frequency is given by

$$
f_{\max } \approx \frac{1.8 c_{L 1}}{36\left(H_{1}+H_{2}\right)},
$$

where $c_{L 1}=6100 \mathrm{~m} / \mathrm{s}$ is the longitudinal velocity of the base plate. Substituting $H_{1}=0.024 \mathrm{~m}$ and $H_{2}=0.010 \mathrm{~m}$ into (40) provides $f_{\max } \approx 8970 \mathrm{~Hz}$, under which (1) is valid. Considering that the maximum excitation force of the exciter employed in the experiments is $50 \mathrm{~N}$ and the response amplitude is small when the frequency is greater than $4000 \mathrm{~Hz}$, the frequency range employed in the numerical analyses was 0 $4000 \mathrm{~Hz}$.

As shown in Figure 3, the mean squared velocities of the base plates $\left(v_{1}^{2}\right)$ for the three specimens were coincident below $3000 \mathrm{~Hz}$, which indicates that the decoupling layer provides negligible vibration suppression for the composite coating in that frequency range. However, above $3000 \mathrm{~Hz}$, the value of $v_{1}^{2}$ for DC-002 decreased due to the enhanced reaction force caused by the increased radiation sound power at high frequencies.

Figure 4 compares $v_{1}^{2}$ and $v_{2}^{2}$ for the three specimens, where the difference $v_{1}^{2}-v_{2}^{2}$ reflects the degree of decoupling and vibration isolation efficiency of the decoupling layer, which increases with positively increasing $v_{1}^{2}-v_{2}^{2}$. As can be observed in Figure 4, DC-001 exhibits no vibration isolation ability, DC-002 amplifies the vibration transmission at frequencies above $2500 \mathrm{~Hz}$, which is undesirable, and DC003 demonstrates good vibration isolation performance. The explanation for these results is as follows. The decoupling layer is essentially a soft vibration isolation pad, which lessens the sound radiation by reducing vibration transfer from 


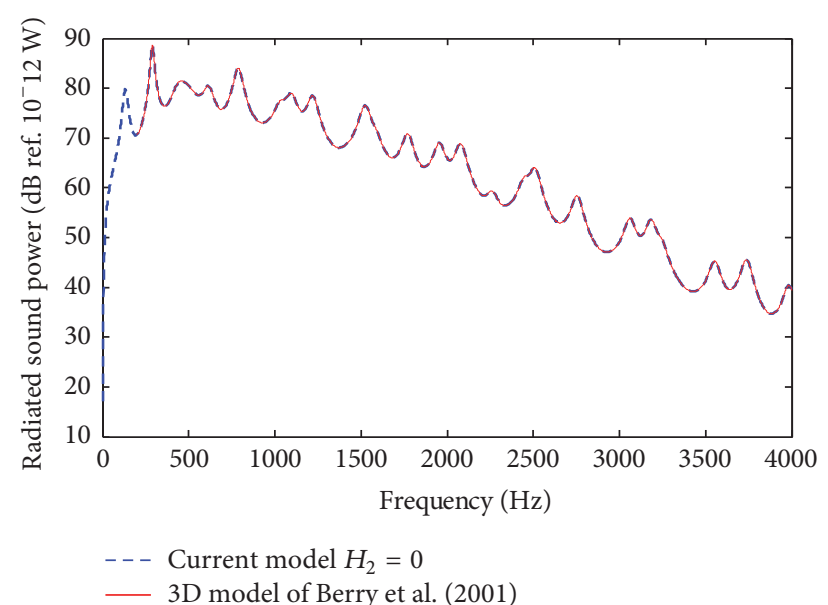

FIGURE 2: Sound power radiated by the system, compared with [17] $\left(H_{1}=9 \mathrm{~mm}, H_{2}=0, H_{3}=50 \mathrm{~mm}, \rho_{3}=600 \mathrm{~kg} / \mathrm{m}^{3}, E_{3}=7.2(1+\right.$ $0.65 i) \times 10^{5} \mathrm{~Pa}$, and $\left.\sigma_{3}=0.38\right)$.

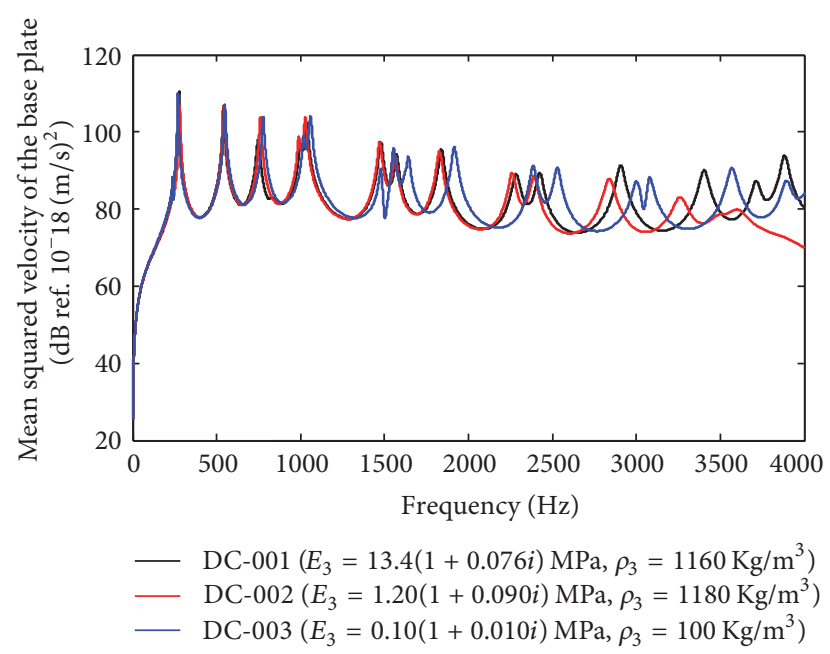

Figure 3: Mean squared velocity of the base plate $\left(v_{1}^{2}\right)$ for the three specimens.

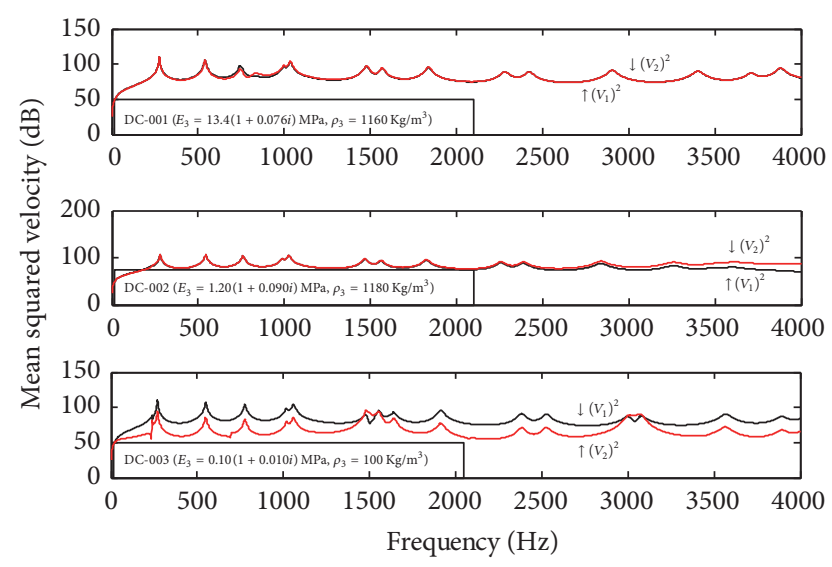

FIgURE 4: Comparison of the mean squared velocities of the base plate and the outer surface $\left(v_{2}^{2}\right)$ of the decoupling layer for the three specimens.
TABLE 1: Structural dimensions and material properties of the composite coating.

\begin{tabular}{lcccc}
\hline Symbol & Units & \multicolumn{3}{c}{ Composite coating } \\
\hline$\rho_{2}$ & $\mathrm{~kg} / \mathrm{m}^{3}$ & 1350 & 1350 & 1350 \\
$E_{2}$ & $\mathrm{~Pa}$ & $101 \times 10^{7}$ & $101 \times 10^{7}$ & $101 \times 10^{7}$ \\
& & $(1+0.97 i)$ & $(1+0.97 i)$ & $(1+0.97 i)$ \\
$\sigma_{2}$ & - & 0.497 & 0.497 & 0.497 \\
$H_{2}$ & $\mathrm{~m}$ & 0.010 & 0.010 & 0.010 \\
$\rho_{3}$ & $\mathrm{~kg} / \mathrm{m}^{3}$ & 1160 & 1180 & 100 \\
$E_{3}$ & $\mathrm{~Pa}$ & $1.34 \times 10^{7}$ & $0.12 \times 10^{7}$ & $1 \times 10^{5}$ \\
$\sigma_{3}$ & - & $(1+0.08 i)$ & $(1+0.09 i)$ & $(1+0.01 i)$ \\
$H_{3}$ & $\mathrm{~m}$ & 0.497 & 0.498 & 0.45 \\
\hline
\end{tabular}

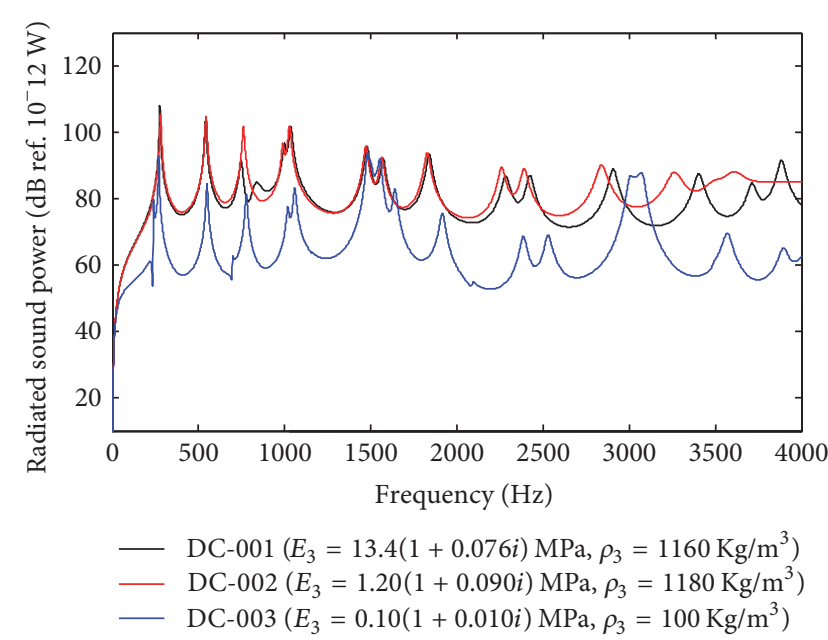

FIGURE 5: Sound power radiated by the three specimens.

the resonant structure to the fluid via vibration isolation. According to (41) [24], when the natural frequency of the decoupling layer is far greater than the excitation frequency, the vibration transmission rate $\left(T_{a}(g)\right)$ of the passive vibration isolation system is close to 1 , and the decoupling layer has no vibration isolation effect. In contrast, when the stiffness of the decoupling layer provides a natural frequency that is close to the excitation frequency, the vibration transmission and the sound radiation will be amplified. Only when the stiffness of the decoupling layer is sufficiently low, providing a natural frequency that is much less than the excitation frequency, will the system exhibit excellent vibration isolation and noise reduction. Consequently, the decoupling material must have low stiffness and high damping:

$$
T_{a}(g)=\left[\frac{1+4 \zeta_{3}^{2} g^{2}}{\left(1-g^{2}\right)^{2}+4 \zeta_{3}^{2} g^{2}}\right], \quad g=\frac{\omega}{\omega_{3 n}} .
$$

Here, $\zeta_{3}$ is the critical damping ratio of the vibration isolation system and $\omega_{3 n}$ is the natural frequency.

As shown in Figure 5, the trend in the sound radiation power is consistent with that of $v_{2}^{2}$, which indicates that 


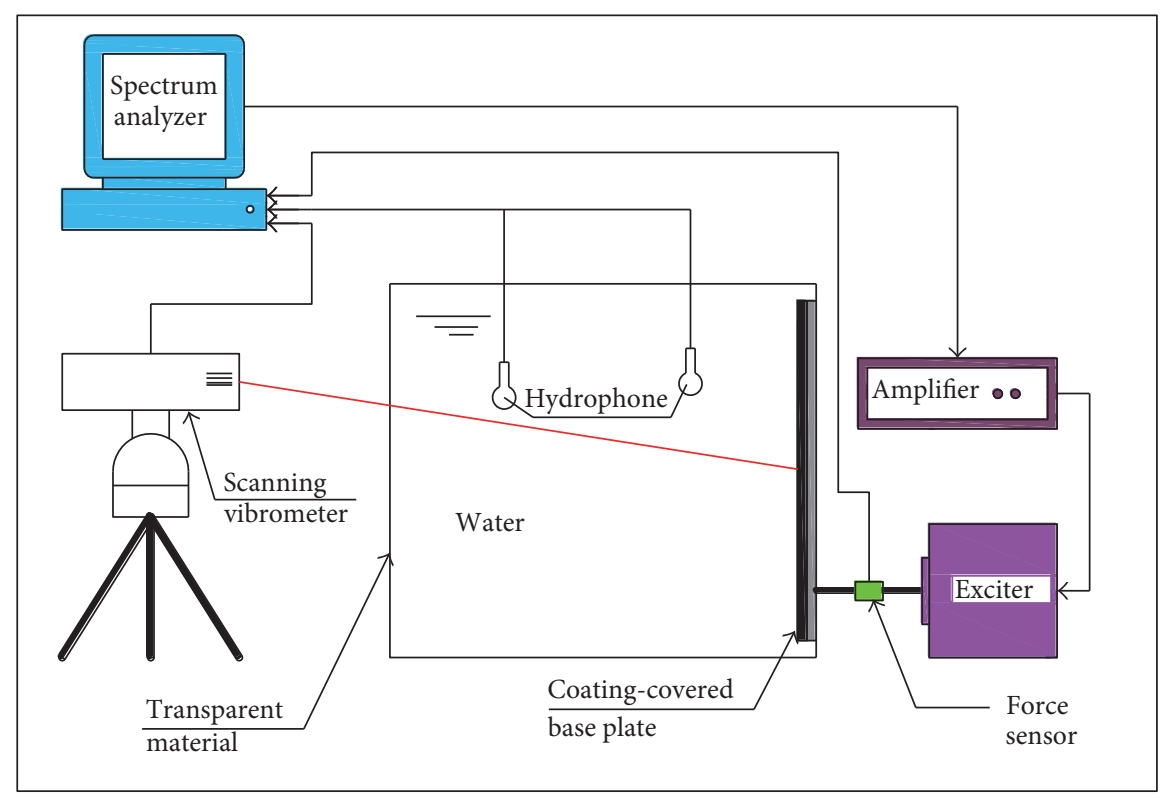

FIGURE 6: Schematic of the experimental test system.

the primary method of noise reduction is to decrease $v_{2}^{2}$. The composite coating employs a damping layer to reduce $v_{1}^{2}$ and applies a decoupling layer to isolate the vibration transmission from the damping layer to the water.

\section{Experiments and Results}

4.1. Description of Experiments. Figure 6 presents a schematic of the experimental setup introduced by $\mathrm{He}$ [11] and Castellini and Santolini [25]. Each test rectangular plate was simply supported on a water tank frame using a $1.0 \mathrm{~mm}$ aluminum enclosure frame and a $5.0 \mathrm{~mm}$ soft rubber gasket. The difference in vibration levels between the tank frame and the test plate was greater than $40 \mathrm{~dB}$. Vibrations were applied by an exciter derived using a random white noise source, and it, in conjunction with a force sensor for measuring the input excitation, was fixed at the base plate at $x_{0}=0.195 \mathrm{~m}$ and $y_{0}=$ $0.145 \mathrm{~m}$ from the plate corner. A Polytec OFV-056 scanning laser vibrometer was employed to measure the responses of the base plate and the outer surface of the decoupling layer. The setup included 126 test points on the base plate surface and 165 test points on the outer surface of the decoupling layer. A pair of B\&K 8104 hydrophones were employed to measure the sound pressure in the reverberant water tank formed by multiple reflections of sound radiating from the internal tank wall surfaces. The water tank included 60 test points, and the results were the mean squared sound pressure based on all 60 test points. Because the sound pressure results were based on multiple reflections, the mean squared sound pressure was only appropriate for qualitative comparison analysis. The materials and dimensions all of the covered test plates were equivalent to those employed in the numerical analyses, as described in Section 3.2.

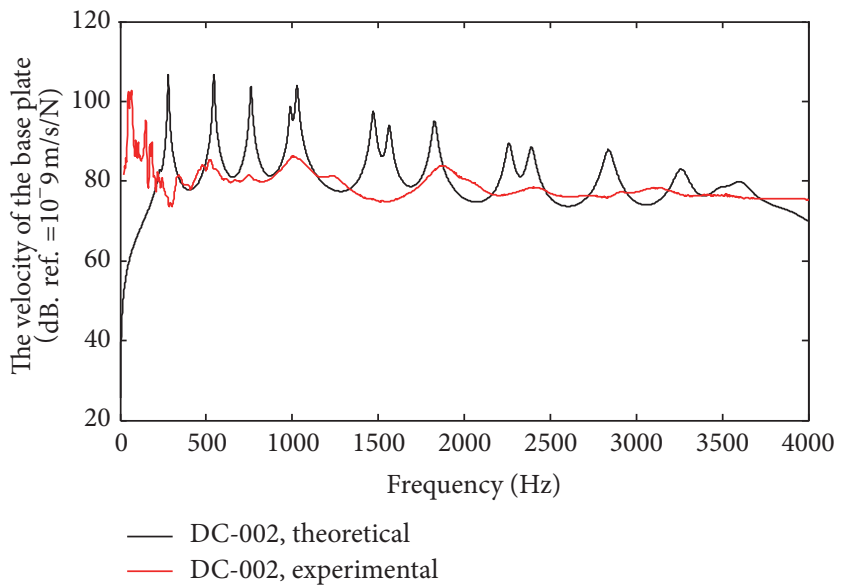

FIgURE 7: Comparison of theoretical and experimental values of $v_{1}^{2}$.

\subsection{Results and Discussion}

4.2.1. Comparison of Theoretical and Experimental Results. Figure 7 shows that the measured values of $v_{1}^{2}$ for DC-002 are essentially equivalent with the numerical results, except that the measured spectral curve is flatter than that of the numerical results, which indicates that the vibration suppression performance of the actual samples was superior to that of the theoretical model. The explanation for this is that the theoretical model ignores the influence of water pressure on the outer surface of the decoupling layer. In fact, the comparisons between measured $v_{1}^{2}$ with and without water in the tank shown in Figure 8 clearly show that water pressure acting on the outer surface of the decoupling layer suppressed the vibrations of the base plate according to (1), particularly in the high frequency range. Second, the numerical analysis did not take into account the frequency dependence of the material parameters. The material parameters employed in the 


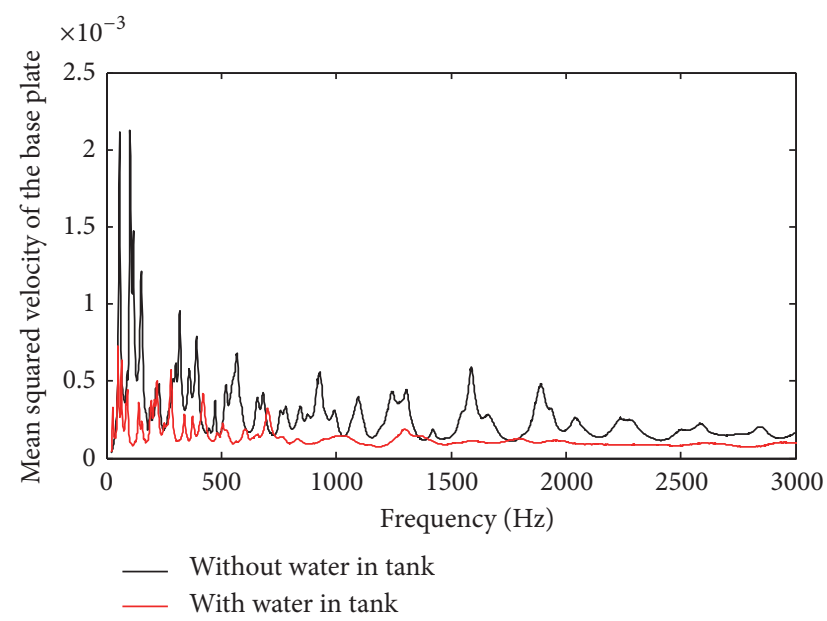

FIGURE 8: Mean squared velocity of the base plate with and without water in the tank.

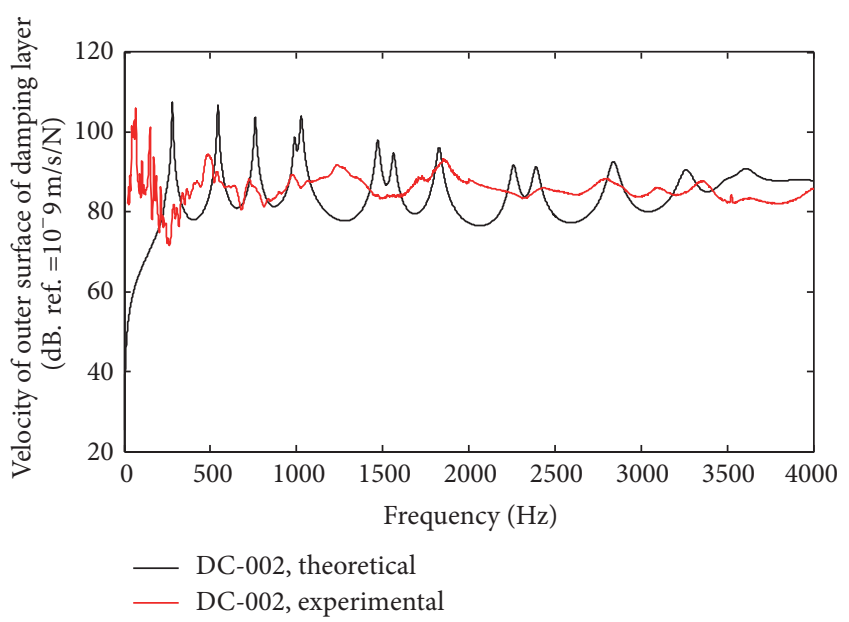

FIGURE 9: Comparison of theoretical and experimental values of $v_{2}^{2}$.

numerical analyses were retrieved from dynamic mechanical analysis (DMA) measurements for the actual samples at $10 \mathrm{~Hz}$ and $16^{\circ} \mathrm{C}$; however, the damping properties of the damping layer material increase with increasing frequency. Finally, the level of the measured reverberant sound pressure is far greater than the radiated sound pressure (Figure 10), resulting in an enhanced reaction force acting on the outer surface of the decoupling layer, which suppressed the vibrations of the base plate according to (1).

As shown in Figure 9, the trend in $v_{2}^{2}$ for DC-002 is roughly in agreement with that of the numerical analysis with the exception of two obvious differences. First, as observed for $v_{1}^{2}$, the measured spectral curve is flatter than that obtained by numerical analysis. Because the role of the decoupling layer is to reduce vibration transfer from the resonant structure to the fluid via vibration isolation, the same three factors affecting the experimental and numerical values of $v_{1}^{2}$ discussed above would be operable here for $v_{2}^{2}$ as well. In addition, as was the case with the damping layer, the numerical calculation neglects that the damping performance of the decoupling material increases with increasing frequency, and, in fact, the resonance peaks become increasingly flattened with increasing frequency. The second obvious difference is that the overall level of the measured $v_{2}^{2}$ is higher than that of the numerical results in the $1100-3200 \mathrm{~Hz}$ frequency band. This is because the frequency range over which the vibration transmission is increased for DC-002 is less than that obtained by numerical analysis (i.e., $2500-4000 \mathrm{~Hz}$ ) due to the influence of the water pressure and the frequency dependence of material parameters.

This is demonstrated in Figure 10, which shows that the trend of the reverberant sound pressure level is consistent with the radiated sound power of the theoretical model but that the level of the former is far greater than that of the latter. As mentioned above, the measured result is the reverberant field of the water tank, and after multiple reflections from the tank wall the radiated sound waves become reverberant sound waves and the level of the sound pressure increases obviously, so this result is only for a qualitative comparison analysis.

4.2.2. Results of the Vibroacoustic Response. Figures 11, 12, and 13 show the measured vibroacoustic responses of samples DC-001 and DC-002. Please note that testing for DC-003 was not implemented.

Figure 11 indicates that the composite coatings demonstrated no vibration suppression effect below $500 \mathrm{~Hz}$. This is because the thickness ratio of the damping layer to the base plate was only 0.42 , which is far less than the engineering experience guidelines of 2.0 [21]. Therefore, conclusions based on test results are provided only for frequencies above $500 \mathrm{~Hz}$. In the $500-2500 \mathrm{~Hz}$ band, the vibration suppression effect of DC-001 is better than that of DC-002, which is attributed to the high loss modulus of DC-002, which is about 10 times greater than that of DC-001. For a free damping structure, the vibration suppression performance increases with increasing loss modulus. In contrast, the vibration suppression effect of DC-001 is no greater than that of DC-002 at frequencies greater than $2500 \mathrm{~Hz}$, which is in accordance with the numerical results, and is caused by the discussed resonance of the decoupling layer.

As shown in Figure 12, the amplification of vibration transmission occurs between 500 and $800 \mathrm{~Hz}$ for DC-001, and the vibration transmission decreases obviously at frequencies greater than $800 \mathrm{~Hz}$. The results are better than those obtained by numerical analysis due to the frequency dependence of the material parameters, as discussed previously. For DC-002, the amplification of the vibration transmission occurs for frequencies greater than $300 \mathrm{~Hz}$. Although noticeable differences between the theoretical and experimental results are observed, the results are generally consistent with the theory of this phenomenon. The differences derive from the fact that the theoretical model ignores the influence of water pressure and the frequency dependence of the material parameters. As can be seen in Figure 13, the radiated sound pressure of DC-002 is greater due to the amplification of the vibration transmission. 


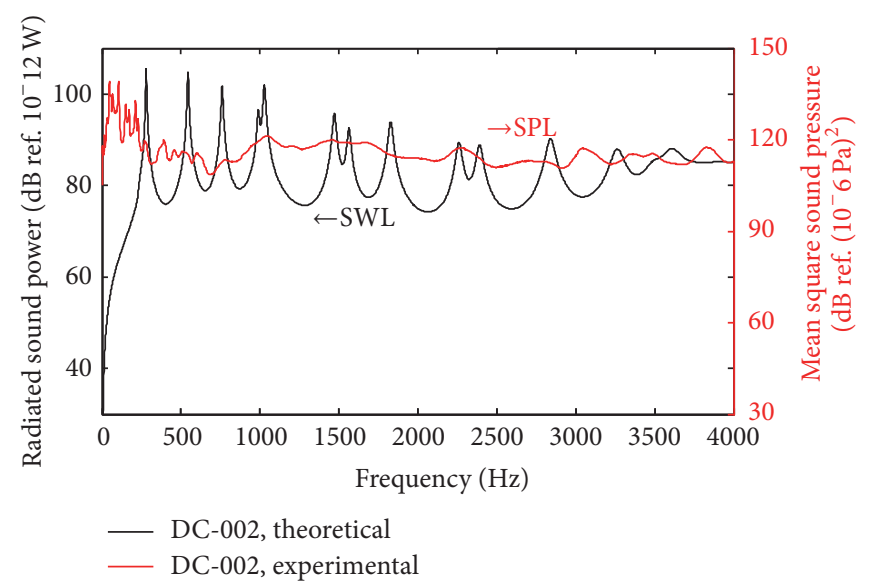

FIGURE 10: Comparison of theoretical and experimental sound fields.

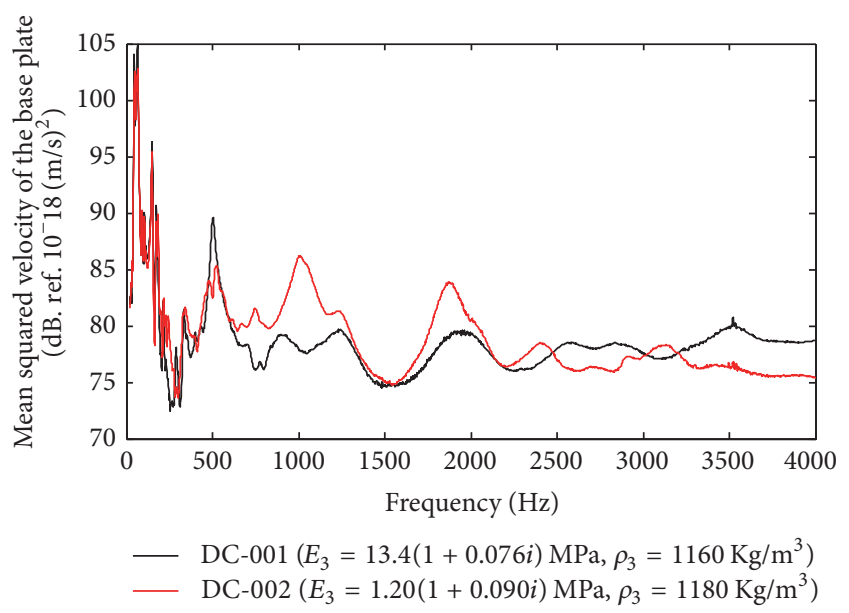

Figure 11: Mean squared velocities of the base plates of two specimens.

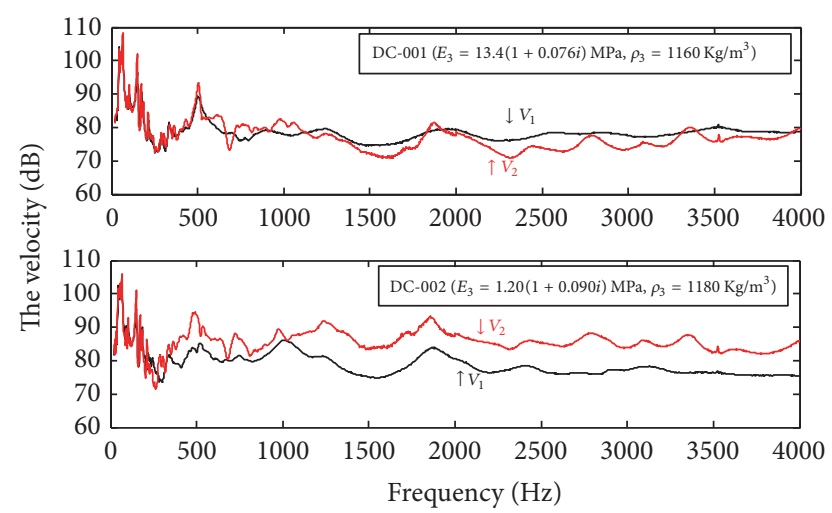

FIGURE 12: Comparison of the mean squared velocities of base plates and outer decoupling layer surfaces.

\section{Conclusions}

This work presented the vibroacoustic response of a fluidloaded, simply supported rectangular plate covered by a damping and decoupling composite acoustic coating.

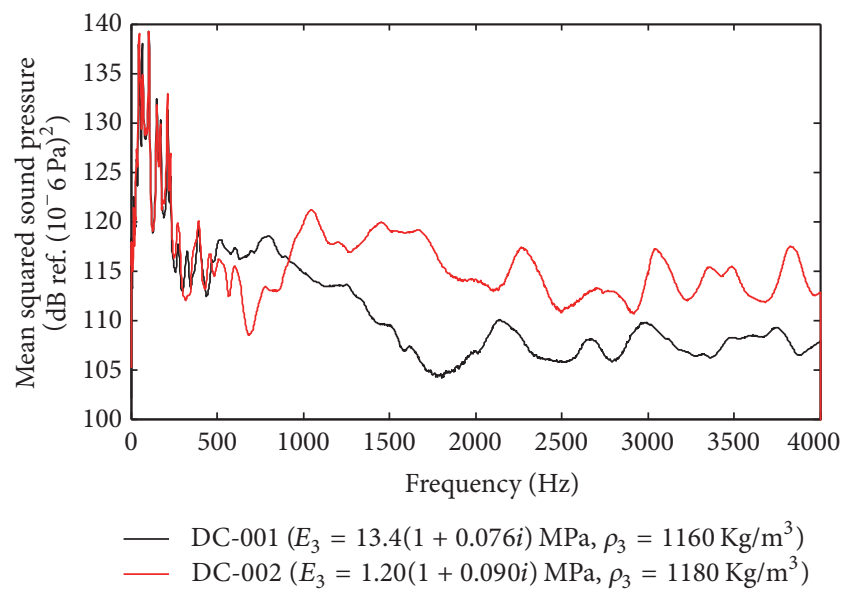

FIGURE 13: Sound pressure radiated by the two specimens.

The vibration suppression performance, decoupling performance, and sound radiation of various composite coatings were studied theoretically and experimentally. Based on the obtained results, the following conclusions can be drawn:

(1) The trends of the theoretical and experimental results are consistent. Some differences are observed due to the influence of water pressure and the frequency dependence of material parameters, which the numerical analysis did not take into account.

(2) The composite acoustic coating combines the merits of both the high vibration suppression performance of a damping coating and the superior vibration isolation performance of a decoupling coating. The radiation noise reduction effect is therefore significantly better than can be obtained using a single-type coating.

(3) Only when the loss modulus of the damping layer is sufficiently high and the stiffness of the decoupling layer is sufficiently low can the system provide an excellent vibration isolation and noise reduction effect. 


\section{Appendix}

\section{Derivation of (36)}

$[A]=\left[\because \frac{a b n \pi}{4}\left\{\left(\rho_{1} H_{1}+\rho_{2} H_{2}\right)\left(\omega_{m n}^{2}-\omega^{2}\right)-\left(\zeta+H_{2}\right)\left[\lambda_{3}\left(\frac{\omega}{c_{L}}\right)^{2}+2 \mu_{3} K_{L, m n}^{2}\right]\right\} \cdot\right]$ (diagonal matrix);

$[B]=\left[\because \frac{a b^{2}}{4} K_{T, m n}\left\{\left(\rho_{1} H_{1}+\rho_{2} H_{2}\right)\left(\omega_{m n}^{2}-\omega^{2}\right)+2\left(\zeta+H_{2}\right) \mu_{3}\left[\left(\frac{m \pi}{a}\right)^{2}+\left(\frac{n \pi}{b}\right)^{2}\right]\right\} \cdot.\right]$ (diagonal matrix);

$[C]=\left[\because \frac{n \pi}{b} \cdot \cdot\right] \quad($ diagonal matrix $)$

$[D]=\left[\because \frac{n \pi}{b} K_{L, m n} \cdot\right]$ (diagonal matrix);

$[E]=\left[\because\left[\left(\frac{m \pi}{a}\right)^{2}+\left(\frac{n \pi}{b}\right)^{2}\right] \cdot\right] \quad$ (diagonal matrix);

$[F]=\left[\because \frac{n \pi}{b} \cdot \ddots\right] \quad$ (diagonal matrix);

$[G]=\left[\because-\left(\zeta+H_{2}\right) \frac{n \pi}{b} K_{L, m n} \quad \cdot\right]$ (diagonal matrix);

$[H]=\left[\because-\left(\zeta+H_{2}\right)\left[\left(\frac{m \pi}{a}\right)^{2}+\left(\frac{n \pi}{b}\right)^{2}\right] \cdot\right] \quad$ (diagonal matrix);

$[I]=\left[\because, K_{T, m n} \cdot\right] \quad$ (diagonal matrix);

$[J]=\left[\because\left[\left(\frac{\omega}{c_{L}}\right)^{2} \lambda_{3}+2 \mu_{3} K_{L, m n}^{2}\right] \cos \left(K_{L, m n} H_{3}\right) \cdot\right]$ (diagonal matrix);

$[K]=\left[\because-\left[\left(\frac{\omega}{c_{L}}\right)^{2} \lambda_{3}+2 \mu_{3} K_{L, m n}^{2}\right] \sin \left(K_{L, m n} H_{3}\right) \cdot\right]$ (diagonal matrix);

$[L]=\left[\because . \frac{2 b}{q \pi} \mu_{3}\left[\left(\frac{p \pi}{a}\right)^{2}+\left(\frac{q \pi}{b}\right)^{2}\right] K_{T, p q} \sin \left(K_{T, p q} H_{3}\right) \cdot\right]+\left[\frac{2 j \omega^{3} \cos \left(K_{T, p q} H_{3}\right)}{a n C_{T}^{2} \pi} Z_{\text {mnpq }}\right]$ (full matrix),

$[M]=\left[\because-\frac{2 b}{q \pi} \mu_{3}\left[\left(\frac{p \pi}{a}\right)^{2}+\left(\frac{q \pi}{b}\right)^{2}\right] K_{T, p q} \cos \left(K_{T, p q} H_{3}\right) \cdot\right]-\left[\frac{2 j \omega^{3} \sin \left(K_{T, p q} H_{3}\right)}{a n C_{T}^{2} \pi} Z_{\text {mnpq }}\right]$ (full matrix),

$[N]=\left[\because .2\left(\frac{n \pi}{b}\right) K_{L, m n} \sin \left(K_{L, m n} H_{3}\right) \cdot\right] \quad$ (diagonal matrix);

$[O]=\left[\because .2\left(\frac{n \pi}{b}\right) K_{L, m n} \cos \left(K_{L, m n} H_{3}\right) \cdot\right]$ (diagonal matrix);

$[P]=\left[\because\left[2\left(\frac{m \pi}{a}\right)^{2}+2\left(\frac{n \pi}{b}\right)^{2}-\left(\frac{\omega}{c_{T}}\right)^{2}\right] \cos \left(K_{T, p q} H_{3}\right) \cdot\right] \quad$ (diagonal matrix);

$[Q]=\left[\because-\left[2\left(\frac{m \pi}{a}\right)^{2}+2\left(\frac{n \pi}{b}\right)^{2}-\left(\frac{\omega}{c_{T}}\right)^{2}\right] \sin \left(K_{T, p q} H_{3}\right) \cdot\right]$ (diagonal matrix);

$[R]=\left[\because \frac{m \pi}{a} \cdot \cdot\right]$ (diagonal matrix);

$[S]=\left[\begin{array}{lll}\ddots & \frac{n \pi}{b} & \ddots\end{array}\right]$ (diagonal matrix);

$\left.[T]=\left[\because \frac{m \pi}{a} \cdot\right]\right]$ (diagonal matrix);

$[U]=\left[\begin{array}{lll}\ddots & \frac{n \pi}{b} & \ddots\end{array}\right]$ (diagonal matrix);

$f_{m n}=F_{0} \sin \left(\frac{m \pi x_{0}}{a}\right) \sin \left(\frac{n \pi y_{0}}{b}\right)$. 


\section{Competing Interests}

The authors declare that they have no competing interests.

\section{References}

[1] N. Alam and N. T. Asnani, "Vibration and damping analysis of a multilayered cylindrical shell, part I: theoretical analysis," AIAA journal, vol. 22, no. 6, pp. 803-810, 1984.

[2] N. Alam, "Vibration and damping analysis of a multilayered cylindrical shells, part II: numerical results," AIAA Journal, vol. 22, no. 7, pp. 975-981, 1984.

[3] B. Laulagnet and J. L. Guyader, "Sound radiation from a finite cylindrical shell covered with a compliant layer," Journal of Vibration and Acoustics, vol. 113, pp. 267-272, 1991.

[4] B. Laulagnet and J. L. Guyader, "Sound radiation from finite cylindrical coated shells, by means of asymptotic expansion of three-dimensional equations for coating," Journal of the Acoustical Society of America, vol. 96, no. 1, pp. 277-286, 1994.

[5] B. Laulagnet and J. L. Guyader, "Sound radiation from finite cylindrical shells, partially covered with longitudinal strips of compliant layer," Journal of Sound and Vibration, vol. 186, no. 5, pp. 723-742, 1995.

[6] C. Saravanan, N. Ganesan, and V. Ramamurti, "Vibration and damping analysis of multilayered fluid filled cylindrical shells with constrained viscoelastic damping using modal strain energy method," Computers and Structures, vol. 75, no. 4, pp. 395-417, 2000.

[7] S. Boily and F. Charron, "The vibroacoustic response of a cylindrical shell structure with viscoelastic and poroelastic materials," Applied Acoustics, vol. 58, no. 2, pp. 131-152, 1999.

[8] M. Ruzzene and A. Baz, "Finite element modeling of vibration and sound radiation from fluid-loaded damped shells," ThinWalled Structures, vol. 36, no. 1, pp. 21-46, 2000.

[9] Brekhovskikh, Wave in a Layered Medium, Academy of Sciences of the USSR, Moscow, Russia, 1957.

[10] M. Tao, W. L. Tang, and H. X. Hua, "Noise reduction analysis of an underwater decoupling layer," Journal of Vibration and Acoustics, Transactions of the ASME, vol. 132, no. 6, Article ID 061006, 2010.

[11] S. P. He, "Development summary report of new acoustic coating layer," Tech. Rep., 2010.

[12] X.-H. Miao, R.-Q. Wang, L. Gu, X.-H. Gao, and X.-L. Yao, "Numerical analysis on the sound insulation performance of decoupling material," Journal of Ship Mechanics, vol. 9, no. 5, pp. 125-131, 2005.

[13] G. Maidanik and A. J. Tucker, "Acoustic properties of coated panels immersed in fluid media," Journal of Sound and Vibration, vol. 34, no. 4, pp. 519-550, 1974.

[14] O. Foin, A. Berry, and J. Szabo, "Acoustic radiation from an elastic baffled rectangular plate covered by a decoupling coating and immersed in a heavy acoustic fluid," Journal of the Acoustical Society of America, vol. 107, no. 5, pp. 2501-2510, 2000.

[15] G. Wang, W.-Y. Li, W. L. Li, and J. Du, "Sound radiation from an elastically restrained plate covered by an acoustic decoupling layer," Mathematical Problems in Engineering, vol. 2015, Article ID 659472, 11 pages, 2015.

[16] D. G. Crighton, "Aspects of the reflexion and free wave properties of a composite panel under fluid loading," Journal of Sound and Vibration, vol. 64, no. 4, pp. 467-474, 1979.
[17] A. Berry, O. Foin, and J. P. Szabo, “Three-dimensional elasticity model for a decoupling coating on a rectangular plate immersed in a heavy fluid," Journal of the Acoustical Society of America, vol. 109, no. 6, pp. 2704-2714, 2001.

[18] S. H. Ko, "Reduction of structure-borne noise using an airvoided elastomer," Journal of the Acoustical Society of America, vol. 101, no. 6, pp. 3306-3312, 1997.

[19] R. F. Keltie, "Signal response of elastically coated plates," Journal of the Acoustical Society of America, vol. 103, no. 4, pp. 1855-1863, 1998.

[20] F. X. Xin, "An exact elasticity model for rib-stiffened plates covered by decoupling acoustic coating layers," Composite Structures, vol. 119, pp. 559-567, 2015.

[21] L. Cremer and M. Heckl, Structure-Borne Sound, Springer, Berlin, Heidelberg, 1988.

[22] J. D. Achenbach, Wave Propagation in Elastic Solids, NorthHolland Publishing, Amsterdam, The Netherlands, 1973.

[23] Z. Y. He, "Vibration and near scattering field of immersed rectangular elastic-viscoelastic composite plate in an underwater sound field (II) - scattering field near by composite plate," Acta Acustica, vol. 11, no. 1, pp. 1-19, 1986.

[24] D. Wenjing, Theory of Reduce the Vibration, Tsinghua University Press, Beijing, China, 1988.

[25] P. Castellini and C. Santolini, "Vibration measurements on blades of a naval propeller rotating in water with tracking laser vibrometer," Measurement: Journal of the International Measurement Confederation, vol. 24, no. 1, pp. 43-54, 1998. 


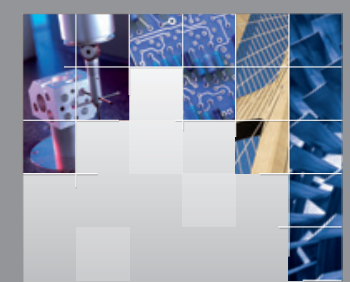

\section{Enfincering}
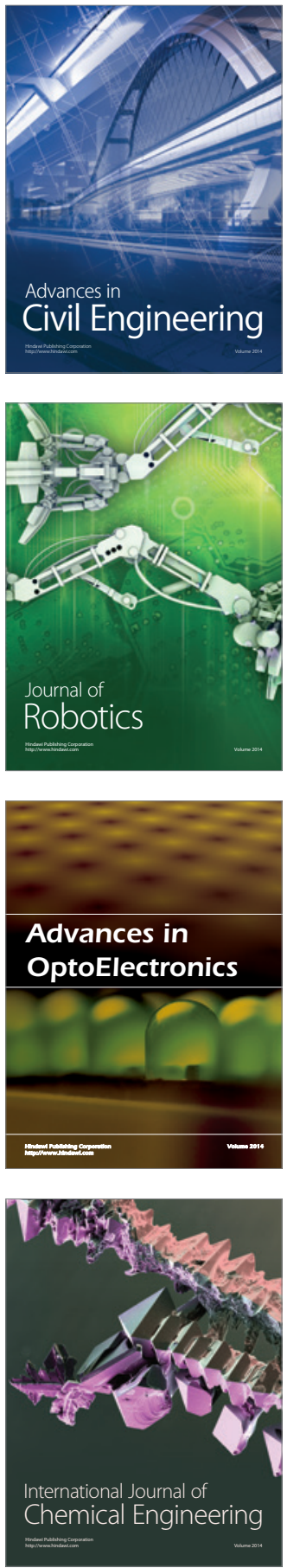

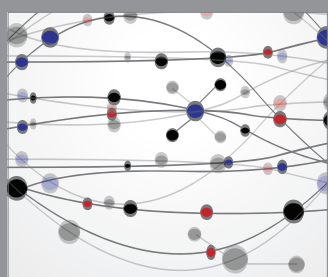

The Scientific World Journal

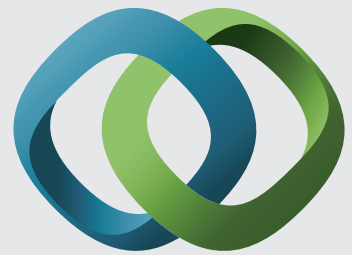

\section{Hindawi}

Submit your manuscripts at

https://www.hindawi.com
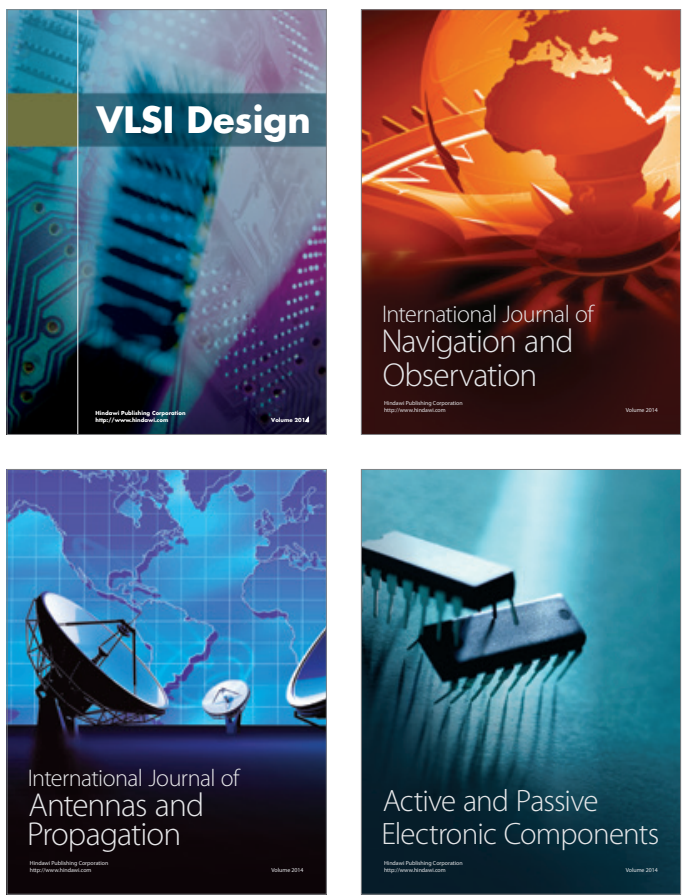
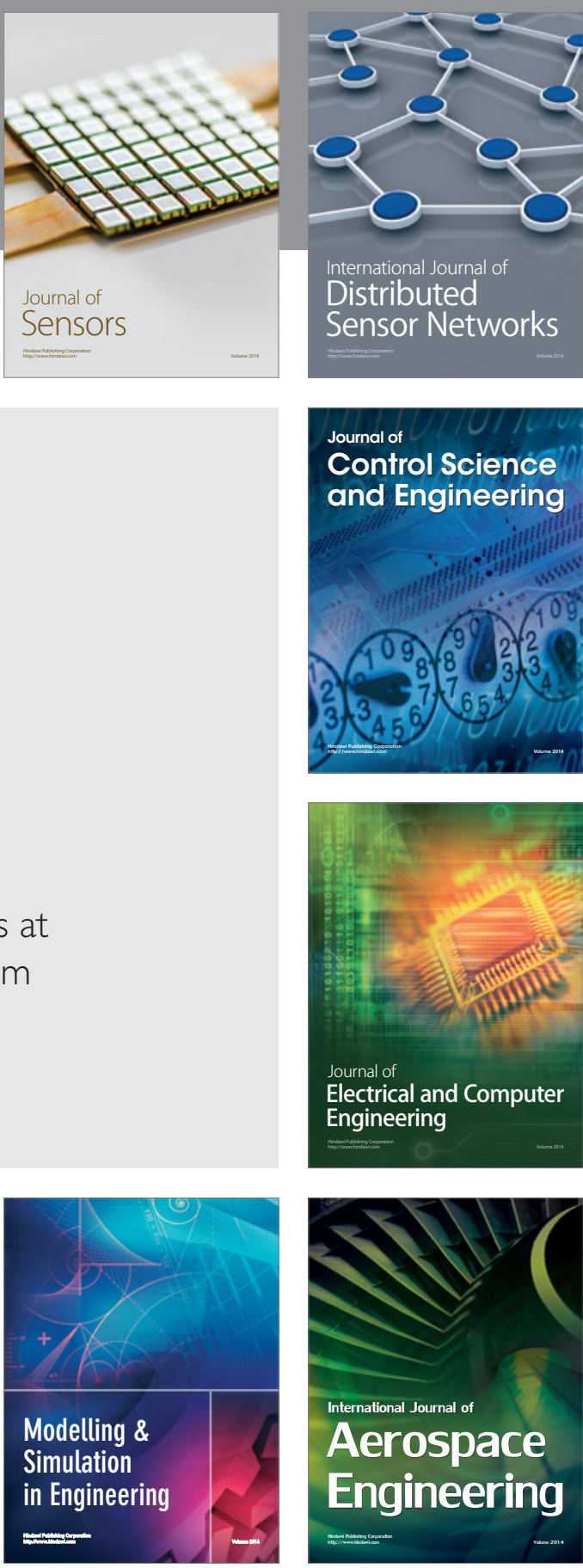

International Journal of

Distributed

Sensor Networks

$-$

Joumal of

Control Science

and Engineering
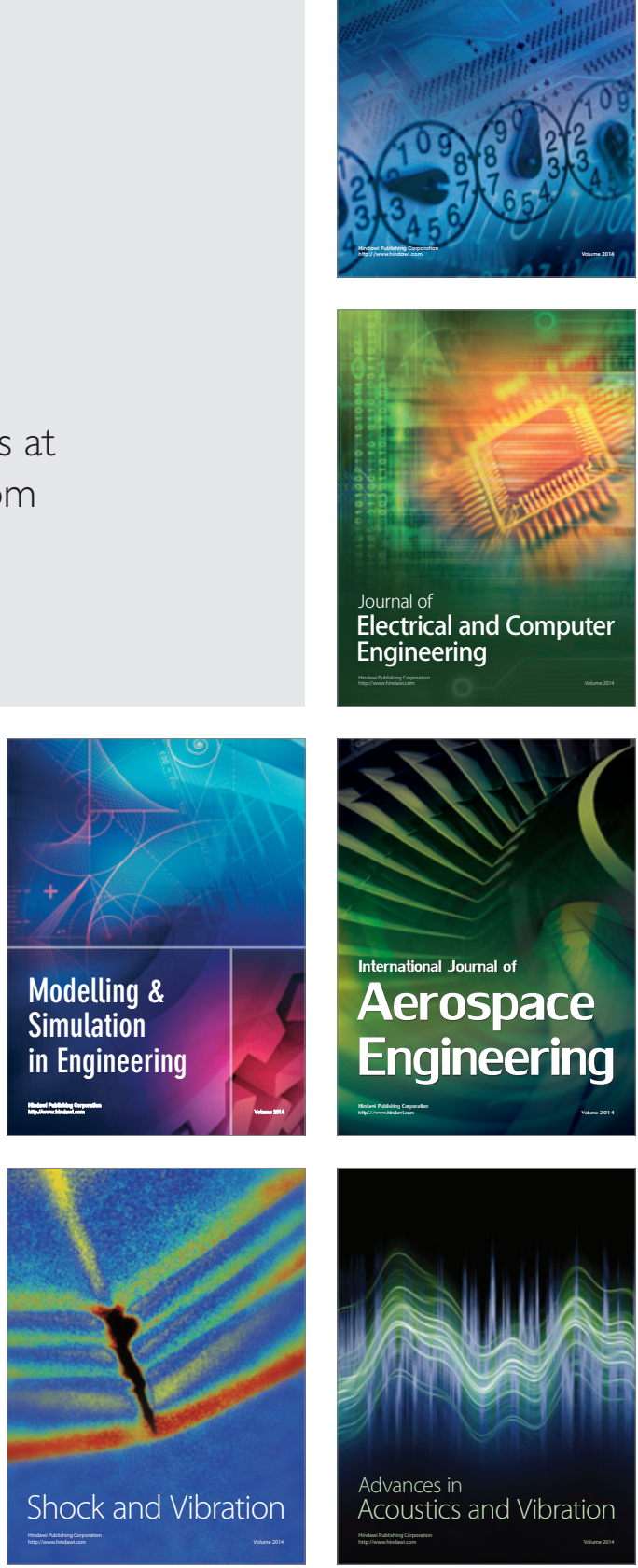